\title{
The Status Quo Theory of Depletion Does not Explain the Israeli Parole Decisions*
}

\author{
Øystein Daljord† Oleg Urminsky ${ }^{\ddagger} \&$ Jose-Manuel Ureta ${ }^{\S}$
}

December 23, 2017

\begin{abstract}
Danziger et al. (2011a) showed that the parole decisions of an Israeli court of parole are influenced by judicially extraneous factors such as the time of day the case is heard. It was suggested in the paper that the cyclical parole frequencies could be explained by a status quo theory of depletion, but it did not test the theory in the data. We first show that the statistical model used in Danziger et al. (2011a) can be interpreted as a behavioral model of a depletion process. Under this interpretation, the status quo theory is inconsistent with the reported results. The statistical model however imposes a restrictive assumption of depletion processes that are uniform across judges. Individual specific depletion processes, which is suggested by theory and supported by the data, affect the measurement of a key test statistic. Allowing for judge specific depletion processes, the evidence is more mixed, but we find overall little support for the status quo theory of depletion.
\end{abstract}

\section{Introduction}

Researchers in the social sciences have long debated the degree to which humans succeed or fail to approximate optimal decisions. A key criterion in this debate has been whether people, particularly trained and experienced experts, are affected by extraneous factors that are normatively unrelated to optimal decisions. In the context of legal reasoning, legal formalists posit that judicial rulings are based exclusively on laws and facts, while judicial realists argue for a role of extra-legal psychological factors, see e.g. Leiter (2005) and Frank (1930).

Danziger et al. (2011a) investigated this question using sentencing data from an Israeli court of parole, documenting a striking pattern in the parole decisions. Its main result is replicated in Figure 1, which plots the relative parole frequency against the order the cases were heard over the course of a day. The parole frequency is strongly cyclical. Within each of three sessions, the initial parole frequency is about $60 \%$, and declines to close to zero by the end of the session. Following a snack break between sessions, the frequency rebounds and then gradually declines again towards the end of the session.

What makes the pattern informative is Danziger et al.'s claim that the ordering of the cases was effectively randomized. Assuming that the case ordering is independent of the case characteristics, the formalism theory, that there is no influence of extraneous factors, makes a sharp prediction: the parole frequency should be constant over the course of a session. As can be seen directly from Figure 1, and as the statistical analysis confirms, the parole frequencies are anything but constant. While Danziger et al. focused on rejecting the null hypothesis of insensitivity to extraneous factors, it also suggested that the declining frequencies within session and the spikes in the frequencies between sessions could be explained by a status quo theory of

\footnotetext{
${ }^{*}$ We thank Jonathan Levav for the data and helpful discussions.

${ }^{\dagger}$ Booth School of Business, University of Chicago, 5807 South Woodlawn Avenue, Chicago, IL 60637, USA. E-mail: oeystein.daljord@chicagobooth.edu

${ }^{\ddagger}$ Booth School of Business, University of Chicago, 5807 South Woodlawn Avenue, Chicago, IL 60637, USA. E-mail: oleg.urminsky@chicagobooth.edu

${ }^{\S}$ Marine Farm, Chile.
} 


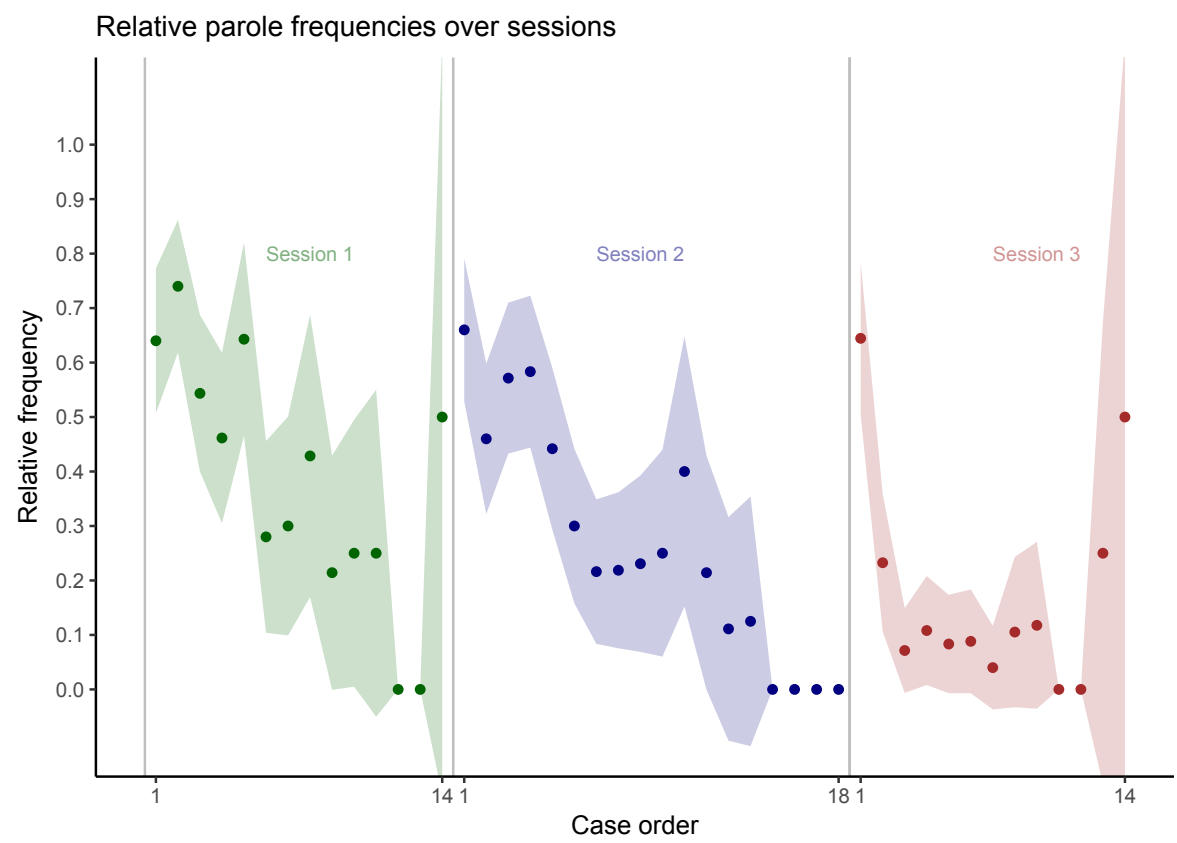

Figure 1: Relative frequencies of granted paroles plotted against case order conditional on at least two observations per case order in each session. The relative frequencies are pooled across judges.

psychological depletion.

While Danziger et al. explicitly did not test this proposed theory, the results have been interpreted as compelling field evidence for biases in expert decision making in a context of high social importance. Kahneman (2011) referred to the paper in his NYT bestseller "Thinking fast and slow" on page 44: "The best possible account of the data provides bad news: tired and hungry judges tend to fall back on the easier default position of denying requests for parole. Both fatigue and hunger probably play a role.".

In this paper, we identify new testable implications of the status quo theory. We introduce a model of sequential decision-making to test these implications using Danziger et al.'s parole data. Our modeling approach is similar in spirit to structural behavioral economics analysis of field experiments (DellaVigna (2017)) in that we state a set of assumptions that map the data distribution to parameters which describe the suggested mechanism.

The status quo theory importantly restricts the permissible range of some of these parameters. While the status quo theory does explain some of the patterns in the data, we find that predictions of the theory are strongly rejected under the original assumptions, which imply depletion processes that are uniform across judges. In subsequent analyses that allow for judge specific depletion processes, the status quo theory is strongly rejected for only a subset of the judges. Overall, we however find little empirical support for the theory. We emphasize that the reason we can test the status quo theory is that it makes a relatively tight range of refutable predictions. We do not test some of the alternative theories, such as fatigue and hunger, as these theories can support a comparatively large a priori possible patterns of outcomes. These alternative theories are therefore hard to reject. We briefly discuss this point in Section 4 .

\subsection{Status Quo Depletion}

A long history of research, going back to the 1980s, has documented systematic effects of fatigue on cognitive performance (Boksem and Tops (2008)). Recently, an influential literature in psychology has proposed that decision-making involves a limited mental resource, which is depleted by making choices, particularly choices requiring self-control (Baumeister et al. (2006), Vohs et al. (2008)). In this view, when the resource is depleted, people will be more likely to make choices that use less of the resource. In particular, this decision process was proposed to be both theoretically and empirically distinct from the effects of prior effort and fatigue 
More specifically, the status quo depletion theory posits that not only is decision making itself cognitively taxing, but changing the status quo (e.g., granting parole) requires more justification, and hence more mental resources, than maintaining the status quo (e.g., denying a parole). Therefore, as a judge is depleted over the course of a decision making session, the judge becomes more likely to deny paroles, either due to lack of mental resources or in a strategic attempt to conserve scarce remaining mental resources (Baumeister (2002)).

According to the theory, depleted mental resources can be replenished by various factors including rest, food intake, and experiences that induce a positive mood (Tyler et al. (2008), Tice et al. (2007), Hagger et al. (2010)). If judges are replenished after a break, they will again be prepared to exert mental effort and make decisions that change the status quo. Subsequently, the judge's mental resources are again depleted by decision making over the course of the sessions and the parole propensity declines. Danziger et al. is careful to note that its data do not include measures directly related to depletion, such as blood sugar levels or a record of what the judges do in the breaks. Still, the paper argues that the breaks the judges take may plausibly be used for activities that are shown to be replenishing in the depletion literature.

The notion of depletion as a meaningful decision making concept has lately been called into question (Inzlicht et al. (2014)). Recent meta-analytic reviews of lab-based tests of depletion have concluded that the effects are small and weak (Carter et al. (2015)). A recent high-powered pre-registered replication largely failed to replicate the lab-based findings (Hagger et al. (2016)), although there has been debate about the interpretation of this non-replication (Dang et al. (2016)). Given the current state of the literature, Danziger et al.'s evidence suggesting status quo depletion constitutes unique field-based evidence for the theory.

A useful feature of the status quo depletion theory, which has gone unnoticed, is that the set of observable decisions that is consistent with the theory is restricted beyond declining parole propensities and spikes between sessions. The theory also implies that the current decision is influenced by both the number and the kind of decisions made so far in the session. In fact, the most common lab paradigms for testing depletion involve comparing performance in a second task among people who did a task predicted to be less depleting vs. those who did a task predicted to be more depleting.

As applied to the court context, a judge who has just granted a parole should be more depleted, and hence less likely to grant a subsequent parole than that judge would be if he had just denied a parole. According to the status quo depletion theory, the current propensity of parole therefore should depend negatively on the number of paroles granted so far in a session. This prediction is testable and the focus of our analysis in Section 2.2.

\subsection{Analysis of judges' sentencing decisions}

To test for the influence of extraneous factors, Danziger et al. presented both graphical evidence, in its Figure 1, and used a series of logistic regressions to test for statistical significance (its main results are in its Table 1). In particular, the paper finds significant differences by session and by case order within session, as predicted by the status quo depletion theory.

Danziger et al.'s suggestion that depletion explains the decision patterns has been controversial. Under its assumption that the order of cases is effectively randomized, the order and session effects present dramatic evidence for theories of legal realism, and against legal formalism. If the declining parole frequencies are indeed due to depletion, it is a real life example with grave social consequences of a controversial decision-making phenomenon that had previously been mostly demonstrated in the lab.

Moreover, the size of the effects are large. The frequency of paroles drops about 50 percentage points over the course of a session. Glockner (2016) notes that the Cohen's $d$ of 1.96 in Danziger et al. is about 8 times higher than what had previously been found in the literature on depletion, which raises legitimate concerns that the patterns may have other causes. 
One such concern is whether there are case relevant unobserved factors that influenced the case ordering. Weinshall-Margel and Shapard (2011) argues that prisoners without legal representation are typically heard towards the end of a decision session. Weinshall-Margel and Shapard conducts interviews with judges that suggests there may be such systematic patterns in the ordering of cases. If so, the cyclical frequencies may emerge independently of depletion.

Danziger et al. (2011b) report data that do not show a systematic pattern in prisoners' legal representation. It also reports various institutional factors that may effectively randomize the case ordering. Though Danziger et al. (2011b) cannot rule out a systematic ordering of the cases, Section 2.2 shows that time varying factors are only weakly correlated with the observed case characteristics. Under some assumptions, which we discuss in Section B, correlations between the observable characteristics and the extraneous factors are informative about selection bias. We do not find such correlations in the data. Absent direct evidence to the contrary, however, the assumption of randomized case ordering is untestable.

Glockner (2016) offers an alternative account that does not appeal to decision biases. It shows that under a particular set of assumptions on how judges decide when to take breaks can generate declining parole frequencies of similar magnitudes. Though we think there is merit to explore the explanatory power of rational models of decision making, our concern in this paper is not to provide an alternative way of rationalizing the data, but to test a suggested theory. We however briefly discuss alternative accounts in Section 4 .

We agree that the size of the effects in Danziger et al. (2011a) call into question whether depletion is the sole cause. However, rather than discard the theory a priori, our approach is to restrict the analysis to only use the available evidence, taking the theory under consideration as given, and test all predictions of the theory. All our analyses are premised on randomized case ordering. Given randomized case ordering, we test the degree to which the proposed status quo depletion explanation is consistent with the data. Next, we formally define the assumptions that give rise to the decision-making models we take to the data.

\section{An empirical model of status quo depletion}

Danziger et al. use logistic regressions to test for the influence of extraneous factors. These regressions explain the parole decisions for each judge $i$, for each session $s$, and case order within session $t$, by a set of covariates. The summary statistics of the data are given in the appendix.

The covariates can be partitioned into a set of extraneous factors $x$ and a set of case-relevant characteristics observable to the researchers $z$. The extraneous factors $x$ used in Danziger et al. are the case order within the session (caseorder), the cumulative minutes spent in the current session (cumulativeminutes), and the proportion of paroles granted so far in the session (pastparoles). The case-relevant characteristics $z$ include the nationality of the prisoner, the severity of the offense, and whether or not the prisoner had attended a rehabilitation program.

The observed decisions are $y_{i s t} \in\{0,1\}$, parole denied and granted, respectively. The logistic regressions are of the kind

$$
\begin{aligned}
& y_{i s t}^{*}=\alpha_{i}+x_{i s t} \beta_{i}+z_{i s t} \gamma+\epsilon_{i s t} \\
& y_{i s t}= \begin{cases}1 & \text { if } y_{i s t}^{*}>0 \\
0 & \text { otherwise. }\end{cases}
\end{aligned}
$$

where $y_{i s t}^{*}$ is latent index function. The error term $\epsilon_{i s t}$ is a catch-all variable representing all factors that influence the decisions and are unobservable to the researcher. The error term is assumed to be iid extreme value distributed. The parameter $\alpha_{i}$ is a judge-specific fixed effect. The null hypothesis of no influence of extraneous factors is a test of the restriction that $\beta_{i}$, the coefficients on the extraneous factors, are all zero. Danziger et al. (2011a)'s report results under the restriction that these parameters are equal across judges, i.e. $\beta_{i}=\beta$. Its results show that the null is rejected beyond conventional levels, as is evident from Figure 1. 
The statistical model in (1) can be given an explicit behavioural interpretation. Below, we state a set of assumptions on the depletion process that lets us infer the depletion process from the parameters of the logistic regression. To the best of our knowledge, the status quo theory has only been described verbally and has not been specified in a formal model, so we have limited theoretical guidance on some of the substantive assumptions. We discuss these where relevant.

We can think of the model in (1) as representing the decision process of judges who take all factors of the case into account. The parameters $\beta$ and $\gamma$ can be thought of as weights which the judges attach to the observable extraneous and relevant factors, respectively. If the weighted sum of the four factors exceeds a threshold, arbitrarily set equal to zero, the judge grants a parole, otherwise he denies a parole.

The threshold can equivalently be thought of as varying with the extraneous factors $x_{i s t}$, such that a parole is granted if $\alpha_{i}+z_{i s t} \gamma+\epsilon_{i s t}>-x_{i s t} \beta_{i}$. Since the extraneous factors include covariates that vary over the session, the model allows the threshold to vary over the course of a session, consistent with a depletion process.

We next make a set of substantive assumptions that allows us to interpret the parameters as representing a status quo depletion process.

\section{Assumption 1. Each judge has a leniency which is stable over time}

We maintain the fixed effect assumption of Danziger et al. and interpret it as a disposition for leniency that varies across judges, but is stable over time for each judge. The assumption allows the judges' mental resources to be replenished to judge specific levels between sessions. We distinguish between the leniency, which can be thought of as the mental resource capacity, and the depletion process, which can be thought of as mental resource taxation.

Assumption 2. The depletion process depends on the number of decisions made so far in the session, the kind of decisions made so far in the session, and the time spent in the session.

We represent the depletion process by the linear index $\left[\right.$ pastparoles $_{i s t}$, caseorder $_{i s t}$, cumulativeminutes $\left._{i s t}\right] \beta_{i}$. This is similar to the specification in Danziger et al., but we substitute the number of past paroles in session, pastparoles ist $=$ $\sum_{\tau=0}^{t-1} y_{i s \tau}$ in for the proportion of past paroles in session used in Danziger et al. as the function of past decisions. Our parameterization reflects the assumption that depletion is a cumulative, rather than relative, phenomenon. Changing the variable representing past choices has little impact on the results of our tests, but allows for a cleaner interpretation. We discuss the choice further in Section 2.2.

The specifications we use imply substantive restrictions on the depletion process. Only the number of paroles granted so far in the session matter for the current decision, and not the ordering or the time they were granted. This exclusion restriction implies that the mental depletion following a decision is summarized by the decision itself. ${ }^{1}$ While more general depletion processes can be imagined, we adopt a specification as close as possible to Danziger et al.'s that can also parsimoniously accommodate a differential impact of paroles and denials on the mental resources. $^{2}$

Assumption 3. The mental resources are fully replenished between sessions.

Assumption 4. The depletion process is invariant across sessions.

Assumption 3 identifies the initial condition $y_{i s 0}=0$, i.e. there is no systematic carry-over of depletion from decisions in previous sessions. The assumption breaks dependence between sessions, and by extension between days. Assumption 4 allows us to pool across sessions, which

\footnotetext{
${ }^{1}$ Conditioning on the history of case characteristics would require substantially more information to estimate to reasonable levels of precision.

${ }^{2}$ One alternative specification of the process lets recent paroles have a stronger impact on the current decision than past paroles. We have however found little guidance from theory or empirics which can inform this choice. The results are qualitatively fairly robust to such alternative specifications and our chosen specification has the virtue of being more parsimonious.
} 
saves on degrees of freedom.

The two assumptions seem largely consistent with the data. From Figure 1, the empirical parole frequency starts at about the same level and declines at about the same rate for the first two sessions. The last session however seems to start at a lower level and drop off at a steeper rate. The approximation bias that would result if the last session follows a different depletion process and replenishment level may however be balanced by a decrease in variance as we can pool observations across all sessions.

Whereas Danziger et al. uses a more flexible function of caseorder and session to approximate the parole propensity path over the course of the sessions in their main specification, we opt for a more parsimonious specification to accommodate heterogeneity across judges when we have limited degrees of freedom.

Assumption 5. The case ordering is effectively randomized.

We assume that the additively separable unobservable factors $\epsilon_{i s t}$ are drawn from a continuous distribution, independently of the extraneous factors $x_{i s t}$. The conditional independence assumption is implied by the assumption of randomized case ordering and allows us to interpret the marginal effects of the extraneous factors causally. A continuous distribution of $\epsilon$ is a regularity condition. Along with monotonicity, which is implied by additively separability, ensures that the index function can be uniquely recovered from the data up to a scale, i.e. that it is identified. The assumption is satisfied by the extreme value error distribution, which we use in the application.

Note that we do not require $\epsilon$ to be conditionally independent of the observable case characteristics $z$. If the case ordering is randomized, then $z$ are also drawn independently of the extraneous factors $x$. Consistency of $\alpha$ and $\beta$, our parameters of interest, is therefore robust to dependence of $\epsilon$ given $z$, but consistency of the nuisance parameters $\gamma$ is not.

Assumption 6. The judges are myopic.

Assumption 6 prohibits judges from strategically saving resources in expectation of future decisions. This is a substantive assumption that assumes away potentially testable implications of some depletion theories. For instance, Baumeister (2002) argues that part of the reduction in self-control is strategic, such that decision-makers refrain from exercising self-control in order to conserve the limited resource for future decisions. The testable implication of this claim is that people will be less likely to choose the status quo option when they are making their last decision before an opportunity to replenish (e.g., before a break or at the end of the day), as long as they know it is the last decision. The claim has some empirical support. Muraven (1998) reports finding more of a depletion effect (i.e. reduction in self-control) on a second task when participants know a third task is coming, compared with when they are not aware of the third task.

Accommodating strategic self-control requires substantial additional assumptions on how judges form expectations over the future cases and how judges decide to when to end a session. Though there seems to be an uptick in the parole propensity towards the end of first and the third session in Figure 1, these upticks are based on only a handful of observations. Given the limited data and the need for a parsimonious model that can be tested with the available data, we defer consideration of this prediction to future research. Moreover, we do not explicitly account for how judges decide to end a session, which reportedly is at their discretion, but take the number of cases heard in each session as given. Accounting for the choice of when to take a break, while arguably important (Glockner (2016)), substantially complicates the analysis and is deferred to future research.

We estimate two models, corresponding to either of the following two assumptions.

Assumption 7. The depletion processes are uniform across judges.

Assumption 8. The depletion processes are judge specific.

Assumption 7 restricts all judges to find paroles equall depleting and for all judges to find denials equally depleting and imposes the restrictions $\beta_{i}=\beta$. Assumption 8 leaves all depletion process parameters $\beta$ judge specific. We call this the heterogenous specification. 


\section{$2.1 \quad$ Inference}

The empirical model in (1) is an unbalanced panel, binary choice model with lagged dependent variables and fixed effects. Given Assumptions 3 and 4, which ensures independence between sessions, we take the number of judges to be fixed and consider sessions the relevant asymptotic dimension, i.e. a fixed $N$ large $T$ panel. The lagged dependent variables are not strictly exogenous, which creates error correlations within sessions. We therefore cluster the standard errors on the session level.

\section{$2.2 \quad$ Testing the status quo theory}

The status quo theory makes three main predictions: declining parole propensities within session, spikes in the propensities after breaks, and negative influence of past paroles on the current parole propensity. ${ }^{3}$ Collectively, these predictions allow a larger set of outcomes than the null of no extraneous factors, but they still rule out a large set of plausible outcomes as inconsistent with the theory. It is sufficient for one of the three predictions to fail to reject the theory. From Figure 1, the first two predictions are clearly consistent when the data are pooled across judges. It is however hard to detect state dependence graphically.

Danziger et al.'s regression analyses, however include a test of state dependence. The majority of the statistical models reported in Danziger et al. include a variable that records the proportion of past paroles that day. This variable was intended to control for a possible quota of paroles, a decision making phenomenon in which the judge sets a target number of paroles to grant within a session. ${ }^{4}$ However, as a function of past decisions, the variable captures state dependence in the decisions and belongs to the set of testable extraneous factors.

Decisions are state dependent when the current decision is influenced by decisions in the past. Positive state dependence results in runs of similar decisions. An example is a 'hot hand' in basketball. Negative state dependence is the opposite. The 'gamblers fallacy' is an example of perceiving negative state dependence when there is none. Across all 15 reported specifications in Danziger et al., the parameter on the proportion of past paroles is positive and mostly highly statistically significant. Our uniform depletion process specification, which is reported in Section 3 , replicates this finding.

Positive state dependence implies that the more paroles a judge has granted so far in that session, the more likely he is to grant another parole, all else equal..$^{5}$ The qualifier is important. As seen in Figure 1, the parole frequency is clearly declining within session in Figure 1. By "all else equal", we mean that these are runs of similar decisions relative to that declining trend. Hence, positive state dependence is consistent with declining parole propensities, but inconsistent with the status quo theory, which only allows negative state dependence. Therefore, Danziger et al.'s finding of positive state dependence rejects the status quo theory. ${ }^{6}$

We next note that although the theory in Baumeister and Muraven (2000) suggests heterogeneity in depletion processes, the statistical models in Danziger et al. imply depletion processes thtat are uniform across judges. Allowing for heterogeneous depletion processes can address a well-known measurement problem for the state dependence parameter, which is a key test statistic (Heckman (1981)). We therefore estimate a specification that allows for heterogenous depletion processes and discuss the measurement issue further in the next section.

Allowing for heterogenous depletion processes raises two separate issues. Firstly, we must now consider the domain of the theory: does it apply to all judges, or just some, and if the latter,

\footnotetext{
${ }^{3}$ These three predictions may not exhaust restrictions of the status quo theory. Without a precisely stated theory, it is difficult, however, to determine the full range of restrictions.

${ }^{4}$ It is not clear exactly how the proportion of past decisions can capture a target number of paroles.

${ }^{5}$ Danziger et al. notes this fact on pp. 6892: "Regardless of the analysis we conducted, the parameter estimate was positive and significant, suggesting that a judge who made a large proportion of favorable rulings up to a certain point was, in fact, more likely to rule favorably in a subsequent case.", but does not discuss it further.

${ }^{6}$ Chen et al. (2016) finds strong and significant negative state dependence in the sequential decisions of baseball umpires, loan application officers, and asylum court judges. It takes the evidence as consistent with the 'gamblers fallacy', but seemingly does not find evidence of spikes and declines between the sessions.
} 
which ones? On this issue, we find limited guidance from theory. Following Glockner and Betsch (2011), we choose to maximize the empirical content by interpreting the theory as predicting that all judges display a pattern consistent with the theory. This is stronger than typical tests of behavioural theories in the lab where average effects are typically the statistics of interest. We however think that a suggested theory can be as interesting in what it fails to explain as in what it does explain. In the end, we think of rejecting a falsifiable theory as progress, rather than failure, as it helps developing our understanding. ${ }^{7}$

Secondly, the heterogenous specification comes at the cost of degrees of freedom which results in a substantial loss of power relative to the uniform model. We show in Section 3 that the heterogenous specification yields mixed for the status quo theory, but the evidence is also noisy. Though we do test and reject the theory that all judges are subject to status quo depletion, we view these results as more exploratory and display the results for all judges individually.

\subsection{Measuring state dependence}

State dependence is a common feature in panels of choice data in marketing and economics, ranging from demand for consumer goods, e.g. Seetharaman et al. (1999), to employment choices, e.g. Keane and Sauer (2009). An important question in this literature is whether the state dependence is structural or spurious, see e.g. Heckman (1981). Structural state dependence reflects a decision making phenomenon, while spurious state dependence is caused by a failure to account for unobserved heterogeneity, which here means judge specific depletion processes. Thus, whether we can interpret the estimated state dependence as informative about the depletion process depends on whether we can interpret it as structural.

To interpret the state dependence parameter in the regressions in Danziger et al. as structural requires assuming that all judges follow the same depletion process, except for judge specific leniencies. Since the parameters on all the extraneous factors are uniform across judges in Danziger et al.'s main specification, unobserved heterogeneity in the depletion process leads to residuals that are positively correlated over time within judge. This positive correlation in the residuals loads onto the variable of past decisions and shows up as positive state dependence in the model. The estimated state dependence may therefore reflect a failure of the model to account for unobserved heterogeneity in the decision process, which is spurious, rather than then reflect that paroles are more depleting than denials, which is structural. We give an illustration of the phenomenon in Appendix A.

It is generally difficult to distinguish between structural and spurious state dependence in the data. We adopt the approach of Heckman (1981) and Dubé et al. (2010). The idea is simple: while structural state dependence survives in specifications with richer heterogeneity, spurious state dependence may not. We therefore interpret state dependence that remains after controlling for heterogeneity as structural. We report one uniform specification and one heterogenous specification. Both satisfy Assumptions 1 to 6. The uniform model also satisfies Assumption 7. It is similar to the analyses in Danziger et al. and replicates its findings. The heterogenous model satisfies Assumption 8.

\section{Empirical results}

The estimates of the two models are given in Table 1. For the uniform model, the estimated state dependence parameter for nr favprsesssion is 0.36 , notably positive, and for caseorder is -0.42 , both highly significantly different from zero. The results therefore reject the null of no influence of extraneous factors, which replicates the Danziger et al. result. The positive state dependence is also inconsistent with the uniform version of the status quo depletion theory, as it implies that granting paroles is less depleting than denying paroles. Though there is independent variation in minutes spent in session, the marginal effect time of time spent in session is effectively zero, with small standard errors. To conserve degrees of freedom, we do not estimate judge specific effects on minutes spent in session in the heterogenous specification.

\footnotetext{
${ }^{7}$ Indeed, much of the progress in the field of judgment and decision making research has been made by repeatedly rejecting existing falsifiable theories of choice.
} 


\begin{tabular}{|c|c|c|}
\hline & Uniform depletion process & Heterogenous depletion process \\
\hline intercept & $-0.54(0.91)$ & $-0.47(0.99)$ \\
\hline \multicolumn{3}{|l|}{ Extraneous factors } \\
\hline pastparoles & $0.36(0.07)^{* * *}$ & $0.51(0.13)^{* * *}$ \\
\hline caseorder & $-0.42(0.07)^{* * *}$ & $-0.53(0.13)^{* * *}$ \\
\hline cumulativeminutes & $0.00(0.01)$ & $0.01(0.01)$ \\
\hline \multicolumn{3}{|l|}{ Case characteristics } \\
\hline offence & $0.05(0.10)$ & $0.05(0.10)$ \\
\hline imprison & $-0.25(0.06)^{* * *}$ & $-0.24(0.06)^{* * *}$ \\
\hline monthsrv & $-0.00(0.00)$ & $-0.00(0.00)$ \\
\hline rehab & $1.96(0.87)^{*}$ & $2.12(0.85)^{*}$ \\
\hline nationality & $-0.14(0.15)$ & $-0.10(0.15)$ \\
\hline gender & $-0.30(0.33)$ & $-0.41(0.35)$ \\
\hline \multicolumn{3}{|l|}{ Judge specific leniency } \\
\hline judge 2 & $-0.06(0.24)$ & $-0.11(0.58)$ \\
\hline judge 3 & $-0.34(0.28)$ & $0.04(0.50)$ \\
\hline judge 4 & $-0.28(0.24)$ & $-0.05(0.49)$ \\
\hline judge 5 & $-0.12(0.28)$ & $0.92(0.70)$ \\
\hline judge 6 & $0.33(0.29)$ & $-0.03(0.45)$ \\
\hline judge 7 & $0.03(0.29)$ & $0.08(0.54)$ \\
\hline judge 8 & $0.20(0.28)$ & $-1.12(0.48)^{*}$ \\
\hline \multicolumn{3}{|c|}{ Judge specific state dependence } \\
\hline pastparoles judge 2 & & $-0.20(0.23)$ \\
\hline pastparoles judge 3 & & $-0.23(0.25)$ \\
\hline pastparoles judge 4 & & $-0.66(0.37)$ \\
\hline pastparoles judge 5 & & $-0.78(0.62)$ \\
\hline pastparoles judge 6 & & $-0.36(0.37)$ \\
\hline pastparoles judge 7 & & $0.05(0.21)$ \\
\hline pastparoles judge 8 & & $-0.53(0.27)^{*}$ \\
\hline \multicolumn{3}{|c|}{ Judge specific depletion rates } \\
\hline caseorder judge 2 & & $0.11(0.21)$ \\
\hline caseorder judge 3 & & $-0.02(0.17)$ \\
\hline caseorder judge 4 & & $0.15(0.20)$ \\
\hline caseorder judge 5 & & $0.02(0.32)$ \\
\hline caseorder judge 6 & & $0.25(0.19)$ \\
\hline caseorder judge 7 & & $-0.03(0.20)$ \\
\hline caseorder judge 8 & & $0.47(0.17)^{* *}$ \\
\hline Num. obs. & 1112 & 1112 \\
\hline
\end{tabular}

Table 1: Statistical models

In Figure 2 we have plotted error bars for the estimated judge-specific depletion rates and the state dependence parameters from the heterogeneous model. The error bars are overlaid with a $95 \%$ confidence interval for the parameters from the uniform specification (the grey box). Note that the estimated state dependence and depletion rates for all but judge 1 is now the sum of the base parameter and the judge specific parameters in Table 1, e.g. the state dependence parameter for judge 2 is pastparoles + pastparolesjudge 2 .

The judge specific depletion rates are all centered around the value of the pooled parameter from the baseline specification, at -0.42 (the purple line), but with substantial heterogeneity across judges. The fact that the state dependence parameters from the heterogenous specification are centered around 0.17 , down from the precisely estimated value of 0.36 in the uniform specification, suggests that part of the positive state dependence in the uniform specification can be explained by unobserved heterogeneity.

For three of the eight judges (judges 4, 5 and 8), the estimated state dependence parameters are directionally negative, consistent with the status quo theory. The estimates are however imprecise, particularly for judge 4 , and for judge 8 , the parameter is virtually zero. The estimated state dependence parameters are directionally positive for the remaining five judges, which is inconsistent with the theory. Only judges 1 and 7 are significantly different from zero, with nominal $p$-values given as 0.00005 and 0.00048 , respectively.

A test of the null hypothesis of the status quo depletion theory that restricts all judges to 
have non-positive state dependence, i.e. the strong theory, is rejected if at least one judge has a statistically significant positive state dependence. We call this the strong test. This test consists of eight individual tests, one for each judge, so we need to account for multiple comparisons.

The family wise error rate is the probability of rejecting at least one true null hypothesis when conducting multiple tests. With a Bonferroni correction, which is a conservative bound for the family wise error rate, the $1 \%$-critical nominal $p$-value is $\frac{0.01}{8}=0.0013$, which is larger than the test statistics for judges 1 and 7 by a good margin.

It is still not entirely clear which family of tests we consider. If we consider a test of the strong theory as making three predictions for each judge (spikes, declines, and negative state dependence), which gives a total of 24 tests, the Bonferroni correction gives a $1 \%$ critical value at nominal $p$-value $\frac{0.01}{24}=0.0004$. Hence, the strong test rejects at the $1 \%$ level also for this larger family of tests, and still by a good margin, irrespective of whether the prediction of spikes and declines are met in the data.

Instead of formally testing the predictions of declines within sessions and spikes between sessions, we choose a more exploratory analysis of the patterns. While the results from the homogeneous specification show strong evidence of the predicted spikes, the results from the heterogenous specification are more mixed across judges. In Figure 5, the predicted parole propensities from the heterogenous specification are plotted for each judge. Judges 1 to 5 and Judge 7 display declining empirical frequencies within session and spikes between sessions, while Judges 6 and 8 do not display clear evidence of either declines or spikes.

One possibility is that the status quo depletion theory applies to some judges, but not others, resulting in behavioral patterns consistent with depletion at the aggregate level, even if not all judges exhibit status quo depletion individually. For example, extending the "self-control as a muscle metaphor", Baumeister and Muraven (2000) propose that people with more practice or experience may exhibit weaker depletion effects. This predicts that some judges would show declines during sessions, spikes between sessions, and negative state dependence, while other judges would show weak or no evidence for all three patterns.

However, the results from the judge-level analysis provide little support for this possibility. Only two of the judges ( 4 and 5 ) who show clear spikes in Figure 5 also have an estimated negative state dependence. The state dependence parameter is particularly imprecisely estimated for judge 5, which may reflect that we have low power. Judges 1 and 7 show clear spikes and declines, but these are the two judges with more precisely estimated positive state dependence, which point to a decision process incompatible with status quo depletion for these judges.

Judge 6 displays only modest declines and spikes. Judge 8 has a pattern in fact clearly consistent with the null hypothesis of no extraneous factors: close to zero depletion rate, no obvious spikes, and zero state dependence. Perhaps only Judge 4 can be said to display a pattern that meets all three predictions. Overall, the results therefore do not clearly align with the predictions of the status quo theory.

Some caveats are warranted. We emphasize again that as we are running out of degrees of freedom, we are limited in the positive conclusions that can be drawn. Throughout this analysis, we maintain the assumption of effectively randomized case ordering. We cannot test this assumption directly, but we have not found systematic patterns in the observable case characteristics. We discuss some diagnostic tests that are common in the treatment effect literature in the appendix, which show no obvious signs of non-randomness. Still, absence of evidence is, as always, not evidence of absence. It is nevertheless possible that there is some unobserved factor that influences both the order which the case is heard and the judges' decisions and which is uncorrelated with the observable characteristics.

Perhaps asking whether only one mechanism explains the data for all judges is not the relevant question. There may be multiple mechanisms at play. Finally, though we have accounted for multiple dimensions of heterogeneity and have interpreted the state dependence parameters as purely structural, we can not rule out that there is a spurious component to the estimated 
state dependence that reflects residual unobserved heterogeneity.

\section{Alternative accounts}

The status quo depletion theory suggested by Danziger et al. has specificity: it rules out a material subset of the possible outcomes as inconsistent with the theory. It is due to the specificity of the status quo depletion theory that we could test this controversial theory, and at least for the uniform case, clearly reject it. The specificity of the theory allowed us to explore the degree of overlap between theoretical predictions and empirical patterns, rather than merely question the assumptions of the theory.

The specificity comes from the status quo feature of the theory - the assumption that granting parole, as a violation of the status quo, both impacts and is impacted by the degree of depletion. It restricts the parole propensities to not only be declining within sessions, but also rules out positive state dependence. Without the status quo feature, the only restrictions of more general depletion or fatigue theories on the parole decisions is a discontinuity in the parole propensity between sessions, and non-constant propensities within sessions.

These two predictions allow for a substantially wider range of deviations from a constant parole frequency, the null tested by Danziger et al., to be consistent with some depletion-like process. It is therefore in principle harder to reject. Similarly, if we changed the meaning of status quo from keeping a prisoner incarcerated to something else, such as equating status quo with making the same choice as in the previous decision, the theory may again be consistent with the data. Adapting the theory to accommodate the data, particularly after the data are known, however empties the theory of empirical content.

It may very well be that hunger and fatigue, as suggested by Kahneman, can explain the patterns independently of, or perhaps in combination with depletion, without restricting either the sign of the state dependence or the time trend in the parole propensities. It is for example $a$ priori plausible that simply deciding cases, whether or not parole is granted, is tiring. Fatigue has been shown to reduce attention and increase distractability and errors (Boksem and Tops (2008)). If fatigue in turn leads to less deliberation and greater weight on initial impressions (Webster et al. (1996)), fatigue may reduce the parole propensity, which is consistent with the results. However, fatigue could by the same arguments support increasing parole propensities. Meaningful tests of such theories may require additional data on the judges' mental states.

The example shows that finding an alternative theory that rationalizes known data is usually not that hard. As Glockner and Betsch (2011) notes, rationalizing a particular outcome is not the only criterion of a good theory, it should also exclude a large range of outcomes as inconsistent with the theory. One would hope that alternative accounts have the specificity of the status quo theory. Moreover, a persuasive alternative theory would not only explain the known data, but make refutable predictions for a larger class of decision problems than the one at hand, and, in the best case, clearly specify which class of decision problems that is.

\section{Summary}

Danziger et al. provided evidence that extraneous factors impact judges' sentencing decisions, consistent with a theory of judicial realism, rather than formalism. Our re-analyses, including taking heterogeneity into account, agree with this conclusion, under the assumption of randomized case ordering. Our analyses further demonstrate that evidence of sensitivity to extraneous factors is displayed in many, but not all, of the judges.

Danziger et al. further suggested that a status quo theory of depletion can explain the cyclical patterns in the decisions of the Israeli courts of parole. We first explicitly tested the suggested theory under the assumptions in Danziger et al.'s original analysis and found that the suggested theory is strongly rejected, due to evidence for positive state dependence under the assumption of homogeneity in depletion processes across judges. 
However, as unobserved heterogeneity in the depletion processes is known to cause spurious state dependence, we then analyze the decisions allowing for judge specific depletion processes. Accounting for multiple dimensions of depletion process heterogeneity, the evidence does not provide strong support for the theory, either when assuming that all judges are subject to status quo depletion, or when assuming that a subset of judges who exhibit status quo depletion drive the sensitivity to extraneous factors. In fact, only one judge out of eight may be said to display a pattern that is consistent with the status quo theory, whereas two judges display patterns that are strongly inconsistent with the theory. For the remaining judges, we find the evidence is inconclusive when taking all predictions of the theory into account.

We believe our approach demonstrates the usefulness of identifying all the empirical predictions of a theory of decision-making and explicitly specifying the assumptions required to test the theory. In particular, analysis of state dependence is often not conducted or is subject to potentially spurious findings (due to neglected heterogeneity) in research on decision-making (Hutchinson et al. (2000)). We think that that making tight predictions is an under-appreciated quality of theories of decision-making. The stronger a theory restricts the set of plausible outcomes, the more powerful the theory is. Conversely, a theory that can explain every choice may not explain any choice at all.
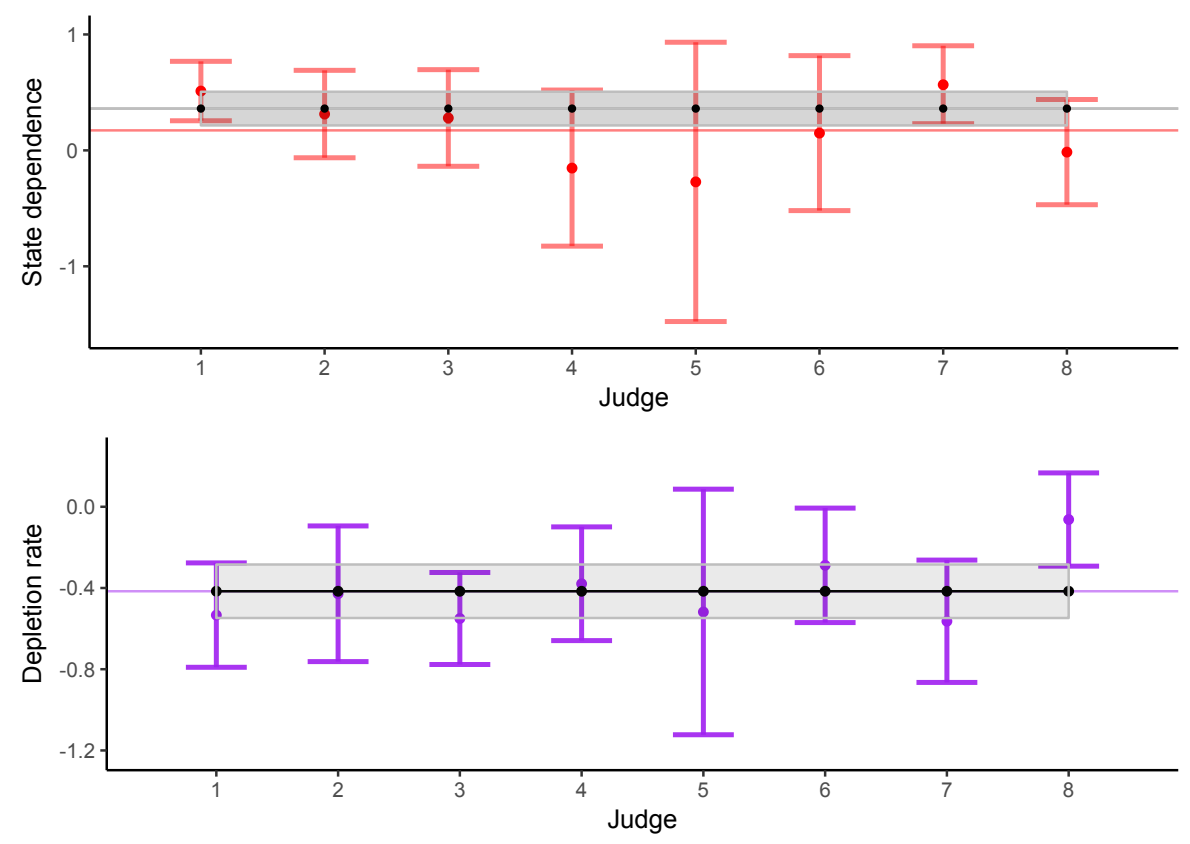

Figure 2: The grey shaded areas in the upper and lower plots give the $95 \%$ confidence interval from the pooled regression. The grey horizontal lines are set at the pooled point estimate. The coloured error bars give the $95 \%$ confidence intervals for the judge specific state dependence and depletion rates in the individual regressions. The coloured horizontal lines give the equally weighted average individual specific parameter.

\section{References}

Altonji, J., T. Eldter, and C. Taber (2005). Selection on observed and unobserved variables: Assessing the effectiveness of catholic schools. Journal of Political Economy 113, 151-184. 15

Baumeister, R. (2002). Ego depletion and self-control failure: An energy model of the self's executive function. Self and identity 2, 129-136. 3, 6

Baumeister, R., M. Gailliot, C. DeWall, and M. Oaten (2006). Self-regulation and personality: how interventions increase regulatory success, and how depletion moderates the effects of traits on behavior. Journal of Personality 74, 1773-1801. 2, 3

Baumeister, R. and M. Muraven (2000). Self-regulation and depletion of limited resources: does self-control resemble a muscle? Psychological Bulletin, 247-59. 7, 10 

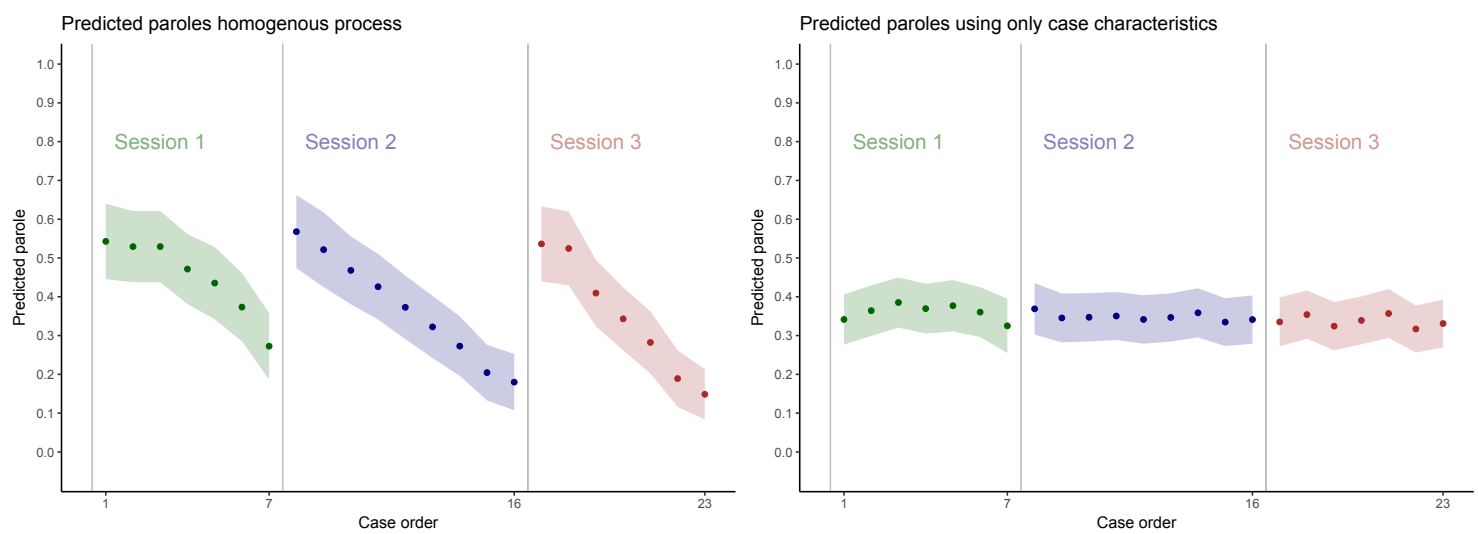

Figure 3: The predicted parole propensities are calculated at the average covariates for the average number of cases per session, assuming uniform depletion processes, using the leniency (fixed effect) of judge 1.

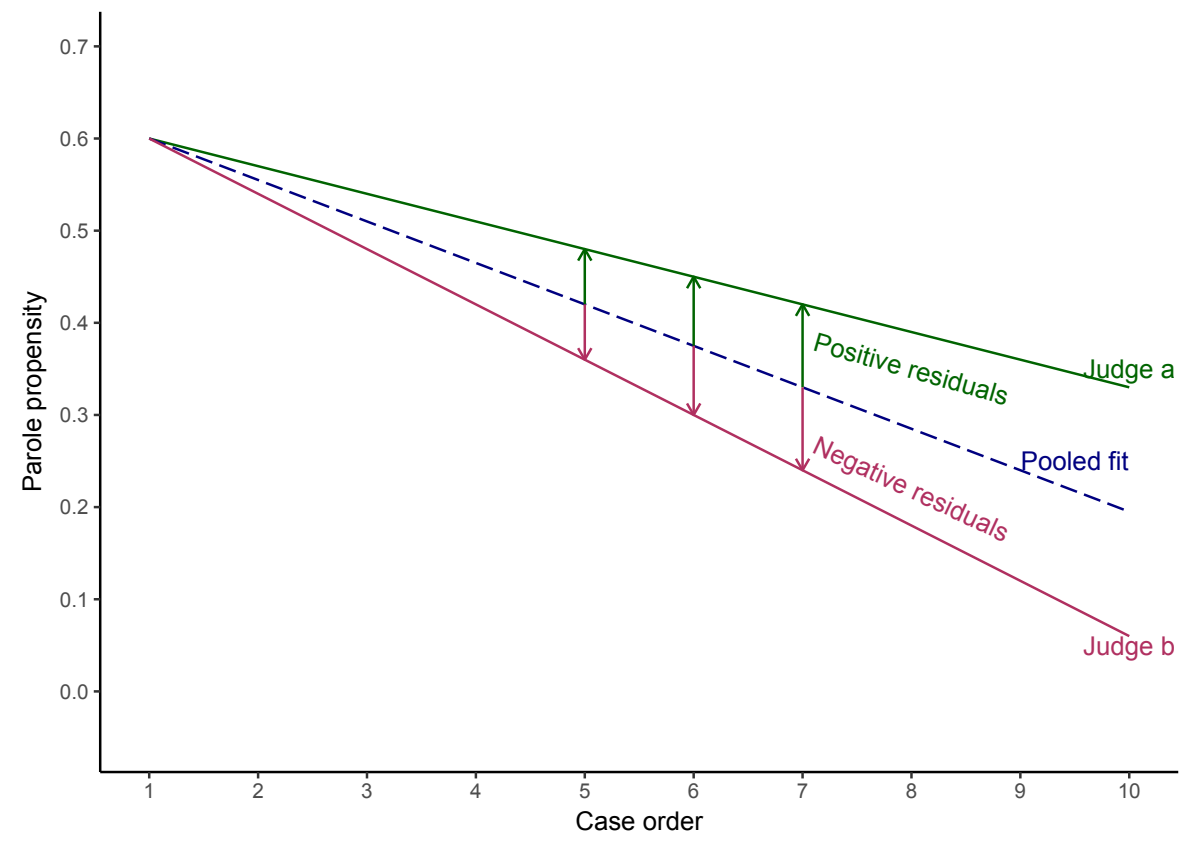

Figure 4: Example of positively correlated model errors resulting from heterogenous depletion processes across judges.

Boksem, M. and M. Tops (2008). Mental fatigue: costs and benefits. Brain Research Review 59, 125-139. 2, 11

Carter, E., L. Koffler, D. Forster, and M. Cullough (2015). A series of meta-analytic tests of the depletion effect: Self-control does not seem to rely on a limited resource. Journal of Experimental Psychology 144, 796-815. 3

Chen, D., T. Moskowitz, and K. Shue (2016). Decision making under the gamblers fallacy: evidence from asylum judges, loan officers, and baseball umpires. Quarterly Journal of Economics 131, 1181-1242. 7

Dang, J., S. Xiao, Y. Liu, and L. Mao (2016). Individual differences in dopamine level modulate the ego depletion effect. International Journal of Psychophysiology 99, 121-124. 3

Danziger, S., J. Levav, and L. Avnim-Pesso (2011a). Extraneous factors in judicial decisions. Proceedings of the National Academy of Sciences USA 108(17), 6889-6892. 1, 2, 3, 4, 5, 6, 7, 8,11

Danziger, S., J. Levav, and L. Avnim-Pesso (2011b). Reply to Weinshall-Margel and Shapard: 
Extraneous factors in judicial decisions persist. Proceedings of the National Academy of Sciences USA 108(42), E834. 4

DellaVigna, S. (2017, November). Structural behavioral economics, Volume 1 of Handbook of behavioral economics. Elsevier. 2

Dubé, J.-P., G. Hitsch, and P. Rossi (2010). State dependence and alternative explanations for consumer inertia. Rand Journal of Economics 41(3), 417-445. 8

Frank, J. (1930). Law and the Modern Mind. New York: Brentano's. 1

Glockner, A. (2016). The irrational hungry judge effect revisited: Simulations reveal that the magnitude of the effect is overestimated. Judgment and Decision Making 11(6), 601-610. 3, 4,6

Glockner, A. and T. Betsch (2011). The empirical content of theories in judgment and decision making. Judgment and Decision Making 6,711-721. 8, 11

Hagger, M., N. Chatzisarantis, H. Alberts, C. Anggono, C. Batailler, A. Birt, R. Brand, M. Brandt, G. Brewer, S. Bruyneel, D. Calvillo, W. Campbell, P. Cannon, M. Carlucci, N. Carruth, T. Cheung, A. Crowell, D. De Ridder, S. Dewitte, M. Elson, J. Evans, B. Fay, B. Fennis, A. Finley, Z. Francis, E. Heise, H. Hoemann, M. Inzlicht, S. Koole, L. Koppel, F. Kroese, F. Lange, K. Lau, B. Lynch, C. Martijn, H. Merckelbach, N. Mills, A. Michirev, A. Miyake, A. E. Mosser, M. Muise, D. Muller, M. Muzi, D. Nalis, R. Nurwanti, H. Otgaar, M. Philipp, P. Primoceri, K. Rentzsch, L. Ringos, C. Schlinkert, B. J. Schmeichel, S. Schoch, M. Schrama, A. Schtz, A. Stamos, G. Tinghog, J. Ullrich, M. vanDellen, S. Wimbarti, W. Wolff, C. Yusainy, O. Zerhouni, and M. Zwienenberg (2016). A multilab preregistered replication of the ego-depletion effect. Perspectives on Psychological Science 11(4), 546-573. 3

Hagger, M., C. Wood, C. Stiff, and N. Chatzisarantis (2010). Ego depletion and the strength model of self-control: a meta-analysis. Psychological Bulletin 136, 495-525. 3

Heckman, J. J. (1981). Statistical models for discrete panel data. In C. Manski and D. McFadden (Eds.), Structural Analysis of Discrete Data with Econometric Applications, pp. 114-178. Cambridge, MA: MIT Press. 7, 8

Hutchinson, W., W. Kamakura, and J. Lynch (2000). Unobserved heterogeneity as an alternative explanation for "reversal" effects in behavioral research. Journal of Consumer Research 27, $324-344.12$

Inzlicht, M., B. Schmeichel, and C. Macrae (2014). Why self-control seems (but may not be) limited. Trends in Cognitive Science 18, 127-133. 3

Kahneman, D. (2011). Thinking Fast and Slow. New York: Farrar, Straus and Giroux. 2, 11

Keane, M. and R. Sauer (2009). Classification error in dynamic discrete choice models: Implications for female labor supply behavior. Econometrica 77, 975991. 8

Leiter, B. (2005). The blackwell guide to philosophy of legal theory. pp. 50-66. 1

Muraven, M. (1998). Mechanisms of self-control failure: Motivation and limited resources. Unpublished doctoral dissertation. 6

Oster, E. (2016). Unobservable selection and coefficient stability. theory and evidence. Working paper, Brown University. 15

Seetharaman, P. B., A. Ainslie, and P. Chintagunta (1999). Investigating household state dependence effects across cateogries. Journal of Marketing Research 36(4), 488-500. 8

Tice, D., R. Baumeister, D. Shmueli, and M. Muraven (2007). Restoring the self: Positive affect helps improve self-regulation following ego depletion. Journal of Experimental Social Psychology 43, 379-384. 3

Tyler, J. M., , and K. C. Burns (2008). After depletion: The replenishment of the self's regulatory resources. Self and Identity 7, 305-321. 3 
Vohs, K., R. Baumeister, B. Schmeichel, J. Twenge, N. Nelson, and D. Tice (2008). Making choices impairs subsequent self-control: a limited-resource account of decision making, selfregulation, and active initiative. Journal of Personality and social psychology 94, 883-898. 2

Webster, D. M., L. Richter, and A. W. Kruglanski (1996). On leaping to conclusions when feeling tired: Mental fatigue effects on impressional primacy. Journal of Experimental Social Psychology 32, 181-195. 11

Weinshall-Margel, K. and J. Shapard (2011). Overlooked factors in the analysis of parole decisions. Proceedings of the National Academy of Sciences USA 108(42), E833. 4

\section{A Example of spurious state dependence}

Suppose two judges $a$ and $b$ both have parole propensities that decline over a decision session, as illustrated in Figure 4. The propensities decline at different rates for the two judges, but by assumption, there is no state dependence for either judge. Suppose next that we pool the observations for both judges and estimate a slope that is uniform across judges. The fit from the pooled regression will be an average of the two slopes.

The residuals from the fitted specification will be autocorrelated, though the model errors are not. The residuals in the example will tend to be positive for judge $a$ and negative for judge $b$, that is, the residuals are positively correlated. The model will underestimate the propensity for judge $a$ (positive residuals) and overestimate the propensity for judge $b$ (negative residuals). In both cases, the variation tends to be absorbed by included functions of past decisions, which gives spurious state dependence.

Similarly, we can note that judge $a$ will grant more paroles than judge $b$ over the course of a session. Judge $a$ therefore has a higher stock of past paroles relative to judge $b$. This higher stock of past paroles is predictive of his future parole propensity, which is higher than the average. Similarly, judge $b$ will grant fewer paroles, and his lower stock of past paroles will explain the negative errors. In both cases, the correlated errors load onto the variable of past decision. In this case, the recovered positive state dependence reflects unobserved heterogeneity in the depletion process, and not a distinct underlying decision making phenomenon.

\section{B Correlations between the extraneous factors and the observable case characteristics}

We do not formally test the assumption of randomized ordering of cases. Instead, we follow a common informal approach, commonly known as the Cambridge Endogeneity Test, which checks if the structural parameters are sensitive to the inclusion of outcome relevant control variables. It is often thought that if the structural parameters are robust to inclusion of relevant control variables, then they are likely also robust to biases from omission of unobserved controls, such as variables that determine the ordering of cases. Though formal tests based on the approach exist, see Altonji et al. (2005) and Oster (2016), we do the informal one to keep it simple.

In Table 2, we have reproduced the uniform model with case characteristics from Table 1 in the first column for convenience. In the second column, we have estimated the uniform model without including the case characteristics. The state dependence and depletion rate parameters hardly move between the two models, though more than one of the observable characteristics are significant. Since adding the observable case relevant factors has little to no impact on the structural parameters of interest, but does significantly improve the fit of the model, one may think there is little selection also on the unobservables.

Finally, we have plotted the predicted probability based on the specification in the third column in Table 2, which conditions on only observable case characteristics on the right hand side of Figure 3. The observable case characteristics are seen to be unable to generate the cyclical patterns in the empirical frequencies compared to the uniform specification on the left hand side, which conditions on the case characteristics and the extraneous factors. 


\begin{tabular}{lccc}
\hline & Uniform with case characteristics & Uniform without case characteristics & Only case characteristics \\
\hline intercept & $-0.54(0.91)$ & $0.67(0.23)^{* *}$ & $-1.64(0.84)$ \\
Extraneous factors & & & \\
$\quad$ pastparoles & $0.36(0.07)^{* * *}$ & $0.36(0.07)^{* * *}$ & \\
$\quad$ caseorder & $-0.42(0.07)^{* * *}$ & $-0.42(0.06)^{* * *}$ & $0.11(0.08)$ \\
$\quad$ cumulativeminutes & $0.00(0.01)$ & & $-0.25(0.05)^{* * *}$ \\
Case characteristics & & & $-0.00(0.00)$ \\
$\quad$ offence & $0.05(0.10)$ & & $1.68(0.76)^{*}$ \\
imprison & $-0.25(0.06)^{* * *}$ & & $-0.05(0.14)$ \\
monthsrv & $-0.00(0.00)$ & & $-0.40(0.28)$ \\
rehab & $1.96(0.87)^{*}$ & & $-0.05(0.20)$ \\
nationality & $-0.14(0.15)$ & $-0.09(0.24)$ & $-0.34(0.28)$ \\
gender & $-0.30(0.33)$ & $-0.37(0.28)$ & $-0.27(0.23)$ \\
Judge specific leniency & $-0.06(0.24)$ & $-0.33(0.24)$ & $0.14(0.28)$ \\
judge 2 & $-0.34(0.28)$ & $-0.15(0.27)$ & $0.54(0.30)$ \\
judge 3 & $-0.28(0.24)$ & $0.31(0.31)$ & $0.10(0.22)$ \\
judge 4 & $-0.12(0.28)$ & $0.02(0.29)$ & $0.14(0.24)$ \\
judge 5 & $0.33(0.29)$ & $0.21(0.29)$ & 1112 \\
judge 6 & $0.03(0.29)$ & 1112 & \\
judge 7 & $0.20(0.28)$ & & \\
judge 8 & 1112 & &
\end{tabular}

Table 2: Statistical models

Table 3: Summary statistics

\begin{tabular}{lccccc}
\hline \hline Statistic & $\mathrm{N}$ & Mean & St. Dev. & Min & Max \\
\hline decision0 & 1,112 & 0.36 & 0.48 & 0 & 1 \\
caseorder & 1,112 & 5.40 & 3.85 & 1 & 25 \\
cumulativeminutes & 1,112 & 27.69 & 26.00 & 0 & 162 \\
pastparoles & 1,112 & 1.97 & 2.05 & 0 & 13 \\
uniquesession & 1,112 & 74.10 & 39.60 & 1 & 145 \\
session & 1,112 & 1.00 & 0.77 & 0 & 2 \\
offence & 1,112 & 2.83 & 0.94 & 0 & 7 \\
imprison & 1,112 & 1.94 & 1.52 & 0 & 13 \\
monthsrv & 1,112 & 28.92 & 34.21 & 0 & 300 \\
nationlty & 1,112 & 0.30 & 0.46 & 0 & 2 \\
gender & 1,112 & 0.95 & 0.22 & 0 & 1 \\
rehab & 1,112 & 0.98 & 0.13 & 0 & 1 \\
judge.d1 & 1,112 & 0.21 & 0.41 & 0 & 1 \\
judge.d2 & 1,112 & 0.20 & 0.40 & 0 & 1 \\
judge.d3 & 1,112 & 0.08 & 0.26 & 0 & 1 \\
judge.d4 & 1,112 & 0.15 & 0.35 & 0 & 1 \\
judge.d5 & 1,112 & 0.07 & 0.25 & 0 & 1 \\
judge.d6 & 1,112 & 0.05 & 0.22 & 0 & 1 \\
judge.d7 & 1,112 & 0.14 & 0.35 & 0 & 1 \\
judge.d8 & 1,112 & 0.11 & 0.31 & 0 & 1 \\
\hline
\end{tabular}



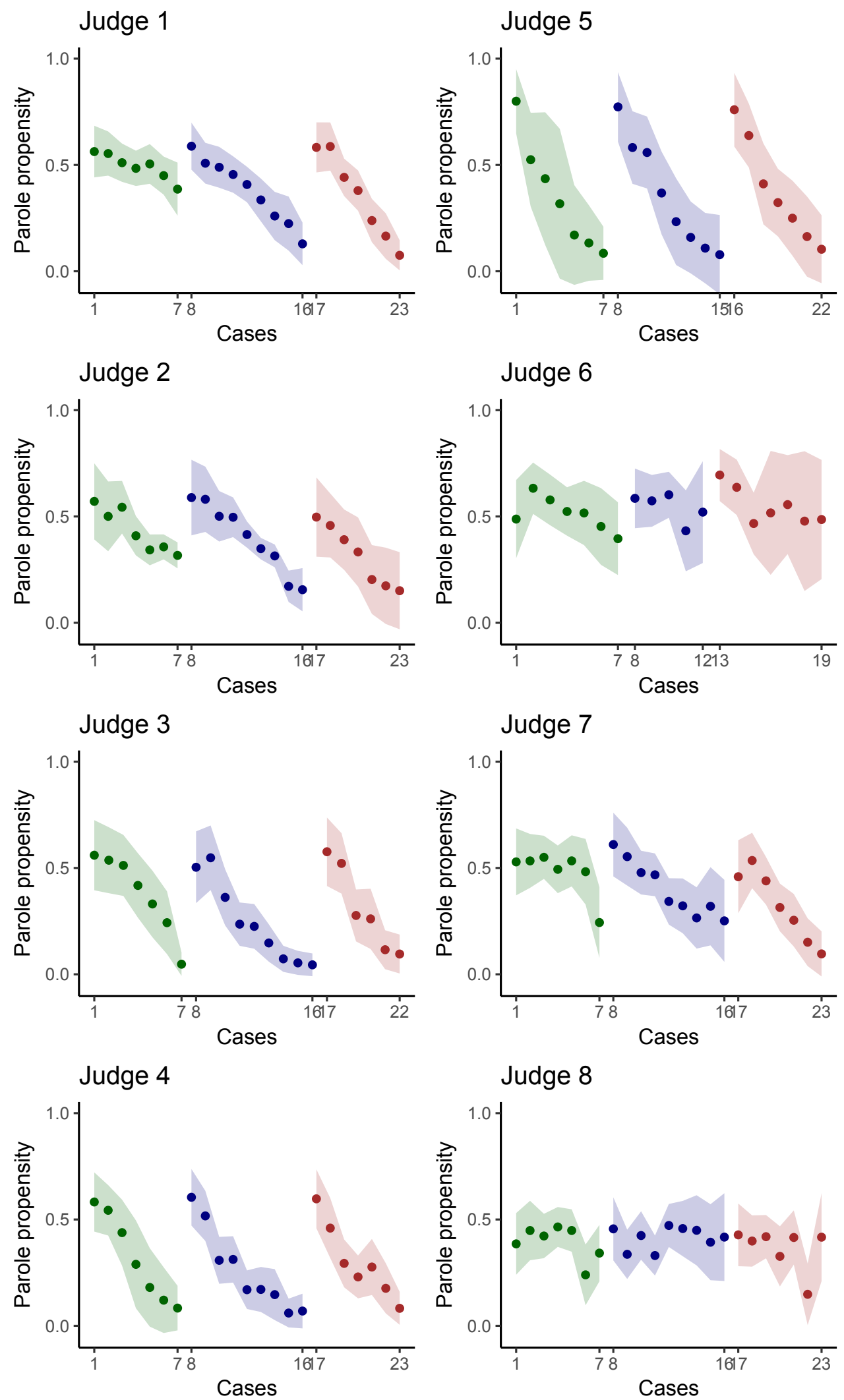

Figure 5: Predicted parole propensities with judge specific depletion rates and state dependence parameters. For each judge, the predicted propensities are calculated at the average covariates and for the average number of cases per session. 